\title{
A practical approach to the patient presenting with dropped head
}

\author{
Marija Cauchi, ${ }^{1}$ Eleanor Marsh ${ }^{2}$
}

\begin{abstract}
${ }^{1}$ Department of Neurosciences, Mater Dei Hospital, Tal-Qroqq, Malta

${ }^{2}$ Department of Neurology, University Hospital of Wales, Cardiff, Wales, UK
\end{abstract}

\section{Correspondence to}

Dr Marija Cauchi, Department of Neurosciences, Mater Dei Hospital, Tal-Qroqq MSD2090, Malta; marijacauchi@gmail.com

Accepted 20 October 2016
To cite: Cauchi M, Marsh $\mathrm{E}$. Pract Neurol 2016;16:445451.

\begin{abstract}
Head drop, or having a dropped head, is an uncommon condition in which patients present with a disabling inability to lift their head. It may arise in many neurological conditions that can be divided into those with neuromuscular weakness of neck extensors and those with increased tone of neck flexors. The most common neuromuscular causes include myasthenia gravis, motor neurone disease and myositis, while neck dystonia secondary to movement disorders can cause an increased tone. Investigations should include blood tests, imaging, muscle biopsy and neurophysiological studies. Here, we present a man with dropped head and discuss the investigation and management of this condition.
\end{abstract}

\section{INTRODUCTION}

The 'dropped head' is uncommon but extremely disabling, with patients having their 'chin on the chest' and unable to extend their head. Patients report the head dropping on to the chest and find great difficulty in seeing where they are going. It can significantly affect activities of daily living and social interaction.

Dropped head can be either neuromuscular or caused by an imbalance in neck muscle tone. Neuromuscular causes are characterised by prominent neck extensor weakness, with minimal or absent neck flexor weakness. It is sometimes described as 'head ptosis' or 'dropped head syndrome'. Somewhat confusingly, the literature also refers to the 'dropped head sign' in the context of cases caused by an imbalance of tone of neck musculature, rather than a primary muscle weakness problem. This includes dystonia of neck flexors, abnormal paraspinal tone and extrapyramidal disorders. Primary skeletal deformities resulting in kyphosis of the upper spine may also present like this. A similar but distinct phenomenon is the forward flexion of the thoracolumbar spine, rather than of the neck in isolation, and this is referred to in the literature by a number of interchangeable terms including camptocormia (ie, kamptō- 'to bend'; kormos - 'trunk'), 'bent spine syndrome' and anteflexion of the spine.

In this article, we focus on the investigation of patients presenting with head drop in the neurology clinic and how to distinguish its possible causes (box 1).

\section{CASE STUDY}

A 56-year-old right-handed man gave a 3 -year history of a subacute stiffness sensation involving his neck. He had been previously well, was working in a garden centre and had been treated for depression with fluoxetine. He became aware that his head would incline forward (having to support his head with his hand) after having been particularly active and on tiring towards the end of the day (figure 1). He noted some difficulty hauling large bags of compost, having previously found such manual heavy lifting relatively easy. For 12 months he had noticed reduced dexterity in the right hand-being unable to apply pressure correctly with a screwdriver. His mother had developed a stooped posture in her $80 \mathrm{~s}$ but there was no other family history of neurological conditions.

On examination, there was wasting and weakness of neck extensors with relatively preserved neck flexors. The neck was easily extended passively. There were moderate neck extensor weakness (MRC (Medical Research Council scale for muscle strength) grade 2/5) and mild neck flexion and shoulder abduction weakness. Otherwise, muscle strength was preserved, with no atrophy or fasciculation. Reflexes were easily elicited in the lower limbs, with no pathological reflexes. There were no sensory signs. Serum creatine kinase was 283 U/L (24-195). Erythrocyte sedimentation rate, serum $\mathrm{C}$ reactive protein, 


\section{Box 1 Causes of dropped head}

Neuromuscular-common

- Isolated neck extensor myopathy

- Motor neurone disease

- Myasthenia gravis (particularly anti-muscle-specific kinase)

- Myositis (polymyositis/dermatomyositis/inclusion body myositis)

- Myotonic dystrophy

Neuromuscular-rare

- Neuropathy, for example, chronic inflammatory demyelinating polyradiculoneuropathy

- Endocrine disorders: hypothyroidism/Cushing's syndrome/hyperparathyroidism

- Carnitine deficiency

- Vitamin $\mathrm{D}$ deficiency

- Drugs for example, dopamine agonists, such as pramipexole

- Post-poliomyelitis syndrome

- Severe hypokalaemic myopathy, for example, liquorice overuse

- Syringomyelia

- Botulism

- Pompe disease

- Oculopharyngeal muscular dystrophy

- Congenital myopathy

- Mitochondrial myopathy

- Hereditary inclusion body myopathy (with valosincontaining protein mutations)

- Mutations in TOR1A/THAP1/C1Z1/GCH1

- Congenital muscular dystrophies with mutations in LMNA

- Facioscapulohumeral muscular dystrophy

Neurodegenerative

- Progressive supranuclear palsy

- Corticobasal degeneration

Abnormal tone

- Idiopathic Parkinson's disease

- Multiple system atrophy

Skeletal

- Osteoporosis

- Ankylosing spondylitis

- Vertebral fracture

serum lactate dehydrogenase, random plasma glucose, $\mathrm{HbA} 1_{\mathrm{C}}, \alpha$-glucosidase concentration, anti-Jo1, serum anti-acetylcholine receptor antibody and anti-muscle-specific kinase (MuSK) antibody were all normal or negative. Neurophysiological assessment 6 months after symptom onset showed normal nerve conduction studies and no clear decrement on repetitive stimulation.

Electromyography (EMG) of the cervical paraspinal muscles showed small amplitude, short duration units (suggesting myopathic units) that were also polyphasic

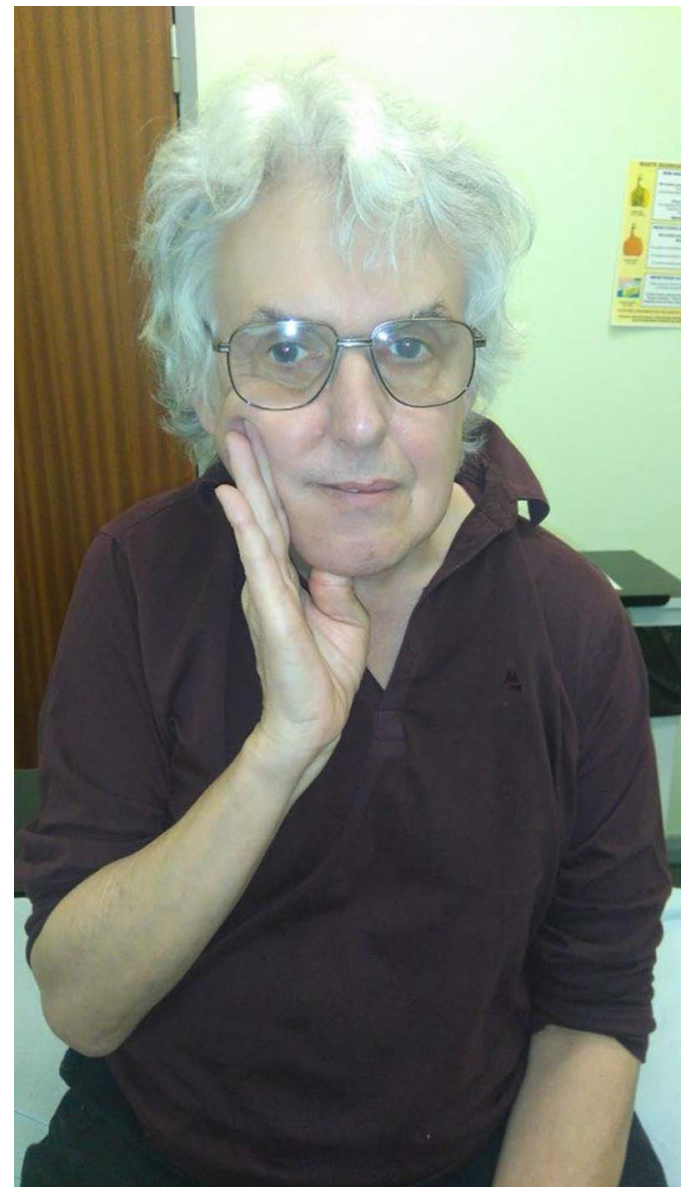

Figure 1 Photograph showing patient with dropped head supporting his chin with his right hand.

(suggesting neurogenic), alongside additional spontaneous activity on testing, that is, a mixed picture in an isolated but clinically relevant muscle. There were no neuropathic or myopathic motor unit action potentials on other muscles tested (deltoid, supraspinatus, infraspinatus, extensor digitorum communis, first dorsal interosseous, quadriceps, biceps femoris). The cervical paraspinal EMG was interpreted as being either focal myopathy or secondary myopathic changes in denervated muscle. MR scan of cervical spine was normal with no evidence of fatty infiltration of the cervical paraspinal muscles.

Muscle biopsy of the trapezius and semispinalis capitis (a cervical paraspinal muscle) 1 year after presentation showed minor non-specific changes in both samples with minor dystrophic changes (some muscle fibres were replaced by connective and adipose tissues) and non-staining cores (of unknown significance). There were small foci of inflammation in semispinalis and the minor changes in trapezius were not considered pathological. He was diagnosed with idiopathic neuromuscular extensor myopathy (isolated neck extensor myopathy) but did not receive corticosteroids as his symptoms were static.

When reassessed 4 years later, he had increasing weakness of shoulder abduction and right first dorsal 
interosseous predominantly, as well as tongue wasting and atrophy. Repeat nerve conduction studies were relatively normal but EMG showed widespread chronic neurogenic changes in the upper limbs as well as brainstem-supplied muscles (genioglossus), in keeping with an anterior horn cell disease. Genetic testing for Kennedy's disease was negative. We altered the diagnosis to a lower motor neurone-predominant form of motor neurone disease. Subsequently he has developed tongue fasciculation with progressive distal intrinsic muscle weakness and has corresponding progressive denervation on neurophysiology, in keeping with this diagnosis.

\section{CLINICAL HISTORY}

Patients generally present with difficulty keeping their head in the neutral position. A detailed history at this stage is important, including the patients' concerns, lifestyle (including job) and limitations.

The age of onset may suggest certain diagnoses. For example, conditions such as inclusion body myositis present in middle age, whereas congenital myopathies may first manifest in adulthood.

The rate of onset and progression also helps: inflammatory conditions occur subacutely, whereas other neuromuscular conditions generally progress more slowly. The patient may not notice the head drop until it is clinically obvious, possibly finding the need to support their chin with a hand or finding that their head drops on to their chest when tired. Some people buy collars for neck support. The strain required to keep their head elevated might cause neck pain. Swallowing might be impaired due to the position of the oesophagus.

Identifying any diurnal variation in symptoms can be important: patients with neuromuscular junction illness such as myasthenia gravis might notice weakness at the end of the day or after exertion, but regain normal strength in the morning. Involvement of other muscle groups might not be evident at presentation but is very important to identify, to distinguish isolated neck extensor myopathy from secondary causes of head drop. Patients might have noticed foot drop (facioscapulohumeral muscular dystrophy/myotonic dystrophy), noticed facial weakness (facioscapulohumeral muscular dystrophy/nemaline myopathy/inclusion body myositis/mitochondrial myopathy/MuSK-positive myasthenia gravis), difficulty swallowing (bulbar weakness in myasthenia gravis/motor neurone disease), ocular weakness (myasthenia gravis/mitochondrial myopathy) or difficulty holding pens or utensils (inclusion body myositis/motor neurone disease/ myasthenia gravis/chronic inflammatory demyelinating polyradiculoneuropathy (CIDP)). Respiratory and diaphragmatic weakness with repeated chest infections can develop in the late stages of several neuromuscular conditions.
A detailed history of other medical problems might provide important clues to the underlying cause. Thyroid or other endocrine disorders can cause neuromuscular weakness. Autonomic symptoms such as postural hypotension in people with neck stiffness could suggest multiple system atrophy. A history of poliomyelitis or vitamin D deficiency may be relevant. Some neurodegenerative conditions, such as progressive supranuclear palsy and idiopathic Parkinson's disease, might present with neck dystonia and so a history of cognitive difficulties is helpful.

Cervical dystonia is an important condition to distinguish from the dropped head syndrome. Patients with cervical dystonia present with subacute onset of hypercontraction of neck flexors, and inability to extend the neck, due to stiffness. The dystonia may be sustained, causing head drop, or repetitive, resulting in rhythmic dystonic tremor. ${ }^{1}$

\section{CLINICAL EXAMINATION}

A detailed neurological examination is essential to identify the pattern of involvement; examination usually enables the clinician to determine whether the cause is abnormal tone or neuromuscular. Weakness of the extensor paraspinal muscles with relative sparing of the flexor muscles implies a neuromuscular cause. In such cases, passive extension of the head should be easy, unless prolonged head drop has led to neck pain or muscle contracture. If the neck can be extended to its normal position only with force, then dystonia of the flexors is more likely. Both neck extensor weakness and flexor muscle dystonia become manifest on standing but subside in a supine/recumbent posture in passive extension (figure 2). ${ }^{2}$ Conversely, skeletal kyphosis is fixed in all positions.

A complete neurological examination is very important to identify the distribution of muscle involvement. Highly selective patterns of weakness in a typical distribution can suggest certain conditions such as inclusion body myositis (wrist and finger flexors, quadriceps). Spontaneous involuntary movements of the muscles, such as fasciculations or myokymia, suggest a lower motor neurone cause. A fasciculating tongue may be present in motor neurone disease. As a rule, most generalised myopathies present with pelvic girdle weakness, causing difficulty climbing stairs or getting out of chairs. These more commonly cause a lumbar lordosis rather than higher spinal anteflexion. Facial weakness is common in hereditary conditions such as mitochondrial myopathy (look for ptosis and ophthalmoplegia) and facioscapulohumeral muscular dystrophy. People with myasthenia gravis commonly develop bilateral ptosis and facial weakness along with weakness of neck extensors and flexors. Testing for fatiguability (myasthenia gravis) or myotonia (myotonic dystrophy) helps to narrow the differential diagnosis. 


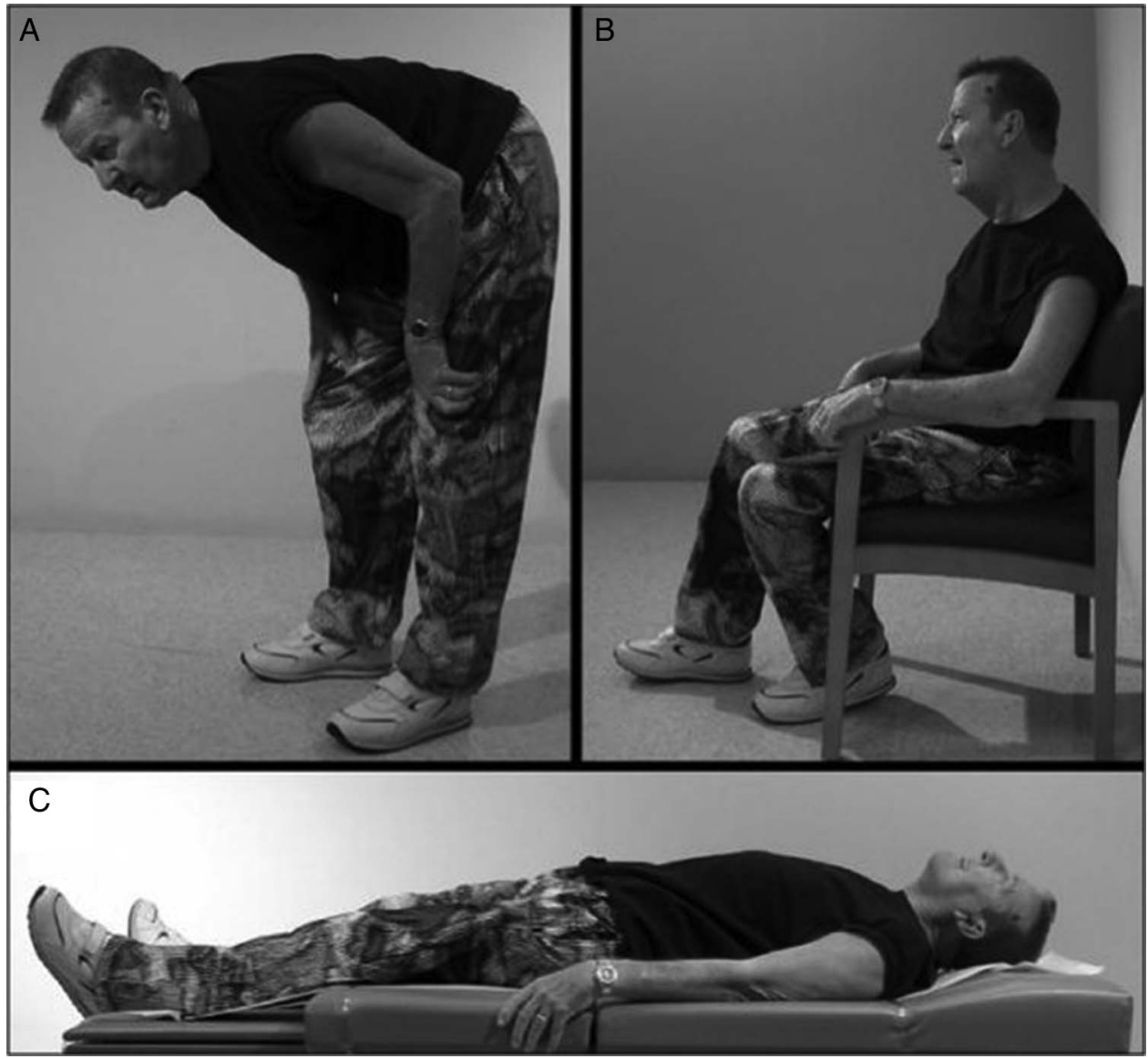

Figure 2 Distinguishing skeletal kyphosis from dystonia or neuromuscular causes of dropped head: the bent spine/neck subsides on laying the patient supine. From Azher and Jankovic. ${ }^{2}$

A general medical examination is also essential. Findings such as organomegaly in polyneuropathy, organomegaly, endocrinopathy, monoclonal gammopathy, and skin changes (POEMS) syndrome or myxoedema in hypothyroidism can also help to identify the cause. Skin examination is particularly relevant when looking for neuromuscular causes of head drop. Dermatomyositis can present with a typical heliotrope rash and Gottron's papules on the hand knuckles; in POEMS syndrome, there can be white nails and finger clubbing.

\section{PRIMARY NEUROMUSCULAR HEAD DROP- ISOLATED NECK EXTENSOR MYOPATHY}

Isolated neck extensor myopathy is a term coined by Katz et al in $1996^{3}$ to describe a restricted form of axial myopathy presenting as an isolated head drop. Despite its name, there is frequently some weakness in other muscle groups (eg, shoulder girdle and upper limbs). It typically presents subacutely in people aged 64-85 years and follows a benign course, with weakness reaching a plateau after 3 months. The neck extension weakness is usually severe and there may also be neck pain. The cause is uncertain, but there is probably a monophasic inflammatory process that resolves spontaneously. ${ }^{3}$ Katz et al also hypothesised that weakness in isolated neck extensor myopathy may follow a loss of tissue elasticity associated with ageing, causing excess stretching of the neck muscles. High blood flow rates in inflammatory myopathies may lead to T-cell-mediated inflammatory conditions specifically affecting the extensor muscles in the neck. ${ }^{4}$ Others suggest that it might be secondary to a neuropathic process causing denervation of the paraspinal muscles in the elderly.

A full investigation of this self-limiting condition should include EMG, which generally shows short duration, low amplitude voluntary motor unit potentials with early recruitment at the cervical paraspinal muscles. This should be interpreted with caution, as cervical paraspinals generally have a small amplitude anyway. Blood tests including serum creatine kinase are usually normal.

It is important to choose the muscles to biopsy carefully. Case reports often describe different biopsy sites, which has led to confusion about its pathological findings. Furthermore, neck muscles are rarely biopsied. Most cases report non-specific myopathic features, with necrotic, moth-eaten and regenerating fibres, myofibrillar disarray, fibrosis and fatty replacement and some have ragged-red fibres. ${ }^{5-8}$ Fibre sizes may be highly variable and nuclei may be centrally 
clustered. Some cases have reported T-cell inflammatory infiltrates, though these cases may be cases of myositis misdiagnosed as isolated neck extensor myopathy. MR scan of muscle (T2 imaging) may show non-specific diffuse oedema of neck extensors.

The management of this condition involves physiotherapy. Patients may require mechanical correction to avoid degenerative changes, such as fixed flexion deformity. This includes using soft or hard collars, semi-braces, halo-braces or surgical fixation. Immunosuppressive agents overall give disappointing results, except where the biopsy shows chronic inflammatory cells. ${ }^{9}$

\section{AXIAL MYOPATHY}

Axial myopathy is another neuromuscular cause of dropped head and camptocormia. In this condition, thoracic kyphosis-due to myopathy of the lower thoracic region-is more prominent than head drop. It presents in middle age, is more common in women and progresses more slowly than isolated neck extensor myopathy. There is sometimes a family history of the condition. Serum creatine kinase might be slightly raised. Biopsies show severe chronic myopathy with fatty change, central nuclei and marked fibre variation. Occasionally there is a focal scant perivascular infiltrate of T-lymphocytes. ${ }^{10}$ MR scan of muscle may show selective and marked involvement of the spinal and neck extensor muscles.

\section{SECONDARY NEUROMUSCULAR CAUSES OF HEAD DROP}

Myopathic illness may initially present as an isolated head drop; it is important to exclude secondary causes before diagnosing isolated neck extensor myopathy.

Myasthenia gravis may rarely present with head drop, especially in MuSK-positive cases and in the elderly. ${ }^{11-12}$ Examination usually identifies weakness of other muscle groups, such as the levator palpebrae superioris, orbicularis oculi and other facial muscles. ${ }^{13}$ The dropped head may be non-fluctuating and nonfatigable. Rarely, the weakness may remain localised to the neck for up to 3 years. ${ }^{14}$ Repetitive nerve stimulation is useful in distinguishing between myasthenia gravis and isolated neck extensor myopathy. ${ }^{15}$

Between $1 \%$ and $2 \%$ of people with motor neurone disease may present with neck or truncal weakness as their first symptom. ${ }^{16}$ Head ptosis may also be a late feature of the disease. EMG helps in its diagnosis, showing a neurogenic pattern with increased spontaneous activity, fibrillations and fasciculation potentials.

Myositis may rarely present in a restricted form, affecting cervical paraspinal and shoulder girdle musculature in isolation. ${ }^{4-5}{ }^{17-18}$ In one case, sporadic inclusion body myositis was restricted to neck musculature and the patient presented with head drop. ${ }^{19}$ Myositis tends to generalise over 3-6 months. Sporadic inclusion body myositis progresses to involve proximal lower limb weakness, with focal atrophy of the quadriceps muscles and distal upper limb weakness. ${ }^{20}$ In such cases, neck involvement usually occurs late in the disease, serum creatine kinase is usually raised and EMG may show a varying pattern of myopathic findings, increased insertional activity and complex repetitive discharges. Biopsies may be similar to those in isolated neck extensor myopathy or may show inflammatory changes.

Other less common causes of head drop include CIDP, which generally involves other muscle groups along with cervical muscles. Head drop may persist for years with little response to treatment. ${ }^{21}$ Nerve conduction studies show a generalised demyelinating polyneuropathy with conduction blocks and prolonged distal latencies and EMG shows a neurogenic pattern. Primary amyloidosis may also cause head drop without involving other muscle groups. ${ }^{22}$

Rare causes of isolated dropped head syndrome include hypokalaemia secondary to excess liquorice intake-found in certain Chinese herbal medications $^{23}$ - and severe but otherwise asymptomatic hypothyroidism. ${ }^{24}$

Certain congenital myopathies, such as those caused by mutations in the LMNA or SEPN1 gene, may rarely present with an isolated head drop, although examination generally shows limb girdle muscular weakness as well as neck weakness. ${ }^{25}$ Mitochondrial myopathies may also present with dropped head: there will also be a raised levels of serum lactate and creatine kinase.

Isolated head drop can occur as a complication of extended field radiotherapy, years after finishing the treatment. Some cases develop muscle weakness up to 30 years later. This can be localised to the irradiated muscle groups; imaging shows diffuse muscular atrophy and EMG shows myopathic changes. ${ }^{26-27}$

Cervical dystonia can develop in parkinsonian syndromes, including idiopathic Parkinson's disease and multiple system atrophy. ${ }^{1}$ This generally presents subacutely over a few weeks and in the late or middle stages of the disease.

\section{INVESTIGATIONS}

See box 2.

\section{BLOOD TESTS}

Serum creatine kinase, if raised, may indicate an inflammatory myopathy, though it can also be significantly raised in other conditions affecting muscles. Serum $\alpha$-glucosidase, easily and rapidly assessed on a blood spot analysis, are usually low in Pompe disease. Box 2 shows other blood tests that may help in the differential.

\section{IMAGING}

Imaging has only limited use in investigating the causes of a dropped head and is very often normal. 


\section{Box 2 Investigations}

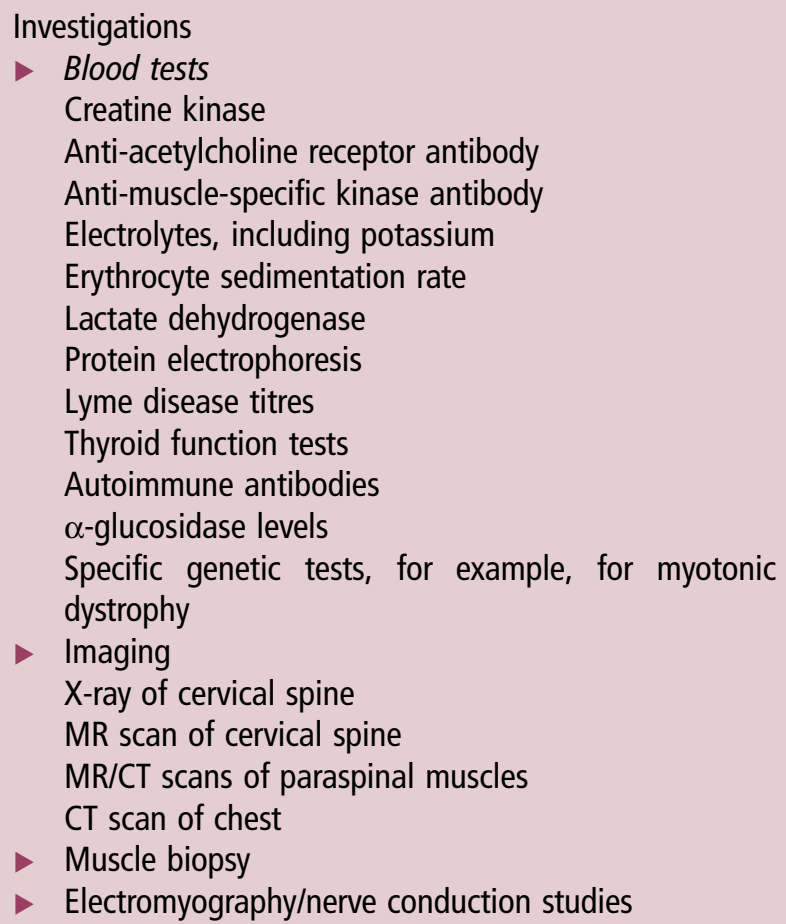

Plain X-ray of the neck can exclude vertebral fractures, osteoporosis and arthritis. MRI or CT scan may show non-specific oedematous or fatty changes with hypertrophy or atrophy of the neck musculature. Gadolinium enhancement on T1 MR imaging can suggest inflammation. ${ }^{3} 28$

\section{BIOPSY}

It is very difficult to interpret muscle biopsies from non-typical biopsy sites, such as neck extensor muscles. It can also be technically challenging to obtain the right biopsy from the neck under image guidance. ${ }^{29}$ The actual yield from neck extensor biopsies is unclear, but interestingly, biopsies of muscles not clinically involved are rarely normal and may show a degree of myopathy, even when the weakness is clinically limited to the neck. Most cases of isolated neck extensor myopathy report atrophic, scattered fibres of varying sizes, with necrotic and regenerating fibres consistent with myopathy. ${ }^{5}$ Perivascular and endomysial inflammatory T-cell infiltrates surrounding necrotic muscle fibres suggest an inflammatory myopathy.

\section{NEUROPHYSIOLOGICAL STUDIES}

Neurophysiological studies are extensively used to help in distinguishing the possible causes of head drop. This is performed on both the paraspinal musculature and the limbs. Findings that suggest certain diagnoses are mentioned in the section on secondary causes of head drop.

\section{OTHER}

Assessing neck extensor muscle strength before and after administering edrophonium could help to confirm the diagnosis of myasthenia gravis.

\section{TREATMENT}

The choice of treatment depends on the primary cause of the dropped head. Corticosteroids and intravenous immunoglobulin may help in myasthenia gravis, CIDP and myositis. Such cases may need prolonged immunosuppression with corticosteroid-sparing agents. Removing the cause (eg, herbal medications) or treating the endocrine condition (eg, hypothyroidism) may lead to resolution of the dropped head.

Surgery may help the cosmetic and physical challenges of head drop, when the patient finds cervical collars either uncomfortable or too distressing. Posterior cervical arthrodesis with fixation from the upper cervical levels to the mid thoracic can correct the head drop though restricts neck rotation. ${ }^{30}$

In summary, the dropped head sign can result from several neurological conditions. Full investigation is important so as not to miss treatable causes. The underlying condition may progress to involve other muscle groups and clinical signs often evolve over time. Consequently, the neurophysiological assessment alters over time. The case described here showed EMG changes consistent with motor neurone disease occurring 4 years after presentation. Muscle biopsy may be unhelpful or show only generalised myopathic changes. Unfortunately, in our case, the diagnosis had to be revised from isolated neck extensor myopathy to motor neurone disease. This emphasises the importance of long-term follow-up and reinvestigation in such patients.

\section{Key points}

- The dropped head ('head on chest') is an uncommon condition, caused by neuromuscular disease (weakness of neck extensor muscles) or increased tone of neck flexors.

- There are several common causes of neuromuscular head drop, with myasthenia gravis, myositis and motor neurone disease being the most common.

- Isolated neck extensor myopathy is diagnosed when there is head drop in isolation with no other identified cause.

- Investigation of head drop includes blood tests, imaging, neurophysiology and biopsy of the muscles involved.

- Treatments, such as immunosuppression, are available for certain causes of dropped head if the cause is identified; conservative management and surgical fixation may be required for unresponsive cases. 
Contributors $\mathrm{MC}$ contributed to the literature review and drafted the article. EM contributed to the conception of the work, literature review and revision of the final draft.

Competing interests None declared.

Provenance and peer review Commissioned; externally peer reviewed. This paper was reviewed by Chris McDermott, Sheffield, UK

\section{REFERENCES}

1 Rivest J, Quinn N, Marsden CD. Dystonia in Parkinson's disease, multiple system atrophy, and progressive supranuclear palsy. Neurology 1990;40:1571-8.

2 Azher SN, Jankovic J. Camptocormia: pathogenesis, classification, and response to therapy. Neurology 2005;65:355-9.

3 Katz JS, Wolfe GI, Burns DK, et al. Isolated neck extensor myopathy: a common cause of dropped head syndrome. Neurology 1996;46:917-21.

4 Kastrup A, Gdynia HJ, Nägele T, et al. Dropped-head syndrome due to steroid responsive focal myositis: a case report and review of the literature. J Neurol Sci 2008;267:162-5.

5 Jaster JH, Bertorini TE, Swims MP, et al. Cervical kyphosis after resolution of myopathic head drop. A case report. Spine (Phila Pa 1976) 1996;21:2023-5.

6 Oerlemans WG, de Visser M. Dropped head syndrome and bent spine syndrome: two separate clinical entities or different manifestations of axial myopathy? J Neurol Neurosurg Psychiatry 1998;65:258-9.

7 Umapathi T, Chaudhry V, Cornblath D, et al. Head drop and camptocormia. J Neurol Neurosurg Psychiatry 2002;73:1-7.

8 Goh KJ, Wong KT, Tan CT. Myopathic dropped head syndrome: a syndrome of mixed aetiology. J Clin Neurosci 2000;7:334-6.

9 Rose MR, Levin KH, Griggs RC. The dropped head plus syndrome: quantitation of response to corticosteroids. Muscle Nerve 1999;22:115-18.

10 Mahjneh I, Marconi G, Paetau A, et al. Axial myopathy--an unrecognised entity. J Neurol 2002;249:730-4.

11 Stickler DE, Massey JM, Sanders DB. MuSK-antibody positive myasthenia gravis: clinical and electrodiagnostic patterns. Clin Neurophysiol 2005;116:2065-8.

12 Tamai M, Hashimoto T, Isobe T, et al. Treatment of myasthenia gravis with dropped head: a report of 2 cases and review of the literature. Neuromuscul Disord 2015;25:429-31.

13 Puruckherr M, Pooyan P, Dube D, et al. The dropped head sign: an unusual presenting feature of myasthenia gravis. Neuromuscul Disord 2004;14:378-9.
14 Casasnovas C, Povedano M, Jaumà S, et al. Musk-antibody positive myasthenia gravis presenting with isolated neck extensor weakness. Neuromuscul Disord 2007;17:544-6.

15 Rodolico C, Messina S, Toscano A, et al. Axial myopathy in myasthenia: a misleading cause of dropped head. Muscle Nerve 2004;29:329-30.

16 Gourie-Devi M, Nalini A, Sandhya S. Early or late appearance of "dropped head syndrome" in amyotrophic lateral sclerosis. J Neurol Neurosurg Psychiatry 2003;74:683-6.

17 Biran I, Cohen O, Diment J, et al. Focal, steroid responsive myositis causing dropped head syndrome. Muscle Nerve 1999;22:769-71.

18 Dominick J, Sheean G, Schleimer J, et al. Response of the dropped head/bent spine syndrome to treatment with intravenous immunoglobulin. Muscle Nerve 2006;33:824-6.

19 Kataoka H, Sugie K, Terashima M, et al. Isolated inflammatory myopathy with rimmed vacuoles presenting with dropped head. Neuromuscul Disord 2009;19:853-5.

20 Hund E, Heckl R, Goebel HH, et al. Inclusion body myositis presenting with isolated erector spinae paresis. Neurology 1995;45:993-4.

21 Hoffman D, Gutmann L. The dropped head syndrome with chronic inflammatory demyelinating polyneuropathy. Muscle Nerve 1994;17:808-10.

22 Chuquilin M, Al-Lozi M. Primary amyloidosis presenting as “dropped head syndrome”. Muscle Nerve 2011;43:905-9.

23 Yoshida S, Takayama Y. Licorice-induced hypokalemia as a treatable cause of dropped head syndrome. Clin Neurol Neurosurg 2003;105:286-7.

24 Askmark H, Olsson Y, Rossitti S. Treatable dropped head syndrome in hypothyroidism. Neurology 2000;55:896-7.

25 D'Amico A, Haliloglu G, Richard P, et al. Two patients with 'Dropped head syndrome' due to mutations in LMNA or SEPN1 genes. Neuromuscul Disord 2005;15:521-4.

26 Rowin J, Cheng G, Lewis SL, et al. Late appearance of dropped head syndrome after radiotherapy for Hodgkin's disease. Muscle Nerve 2006;34:666-9.

27 Furby A, Béhin A, Lefaucheur JP, et al. Late-onset cervicoscapular muscle atrophy and weakness after radiotherapy for Hodgkin disease: a case series. J Neurol Neurosurg Psychiatry 2010;81:101-4.

28 Gaeta M, Mazziotti S, Toscano A, et al. "Dropped-head" syndrome due to isolated myositis of neck extensor muscles: MRI findings. Skeletal Radiol 2006;35:110-12.

29 Waclawik AJ, Lotz BP, Beinlich BR, et al. Dropped head syndrome. Neurology 1997;48:550-1; author reply 551-2.

30 Katirji B, Hachwi R, Al-Shekhlee A, et al. Isolated dropped head due to adult-onset nemaline myopathy treated by posterior fusion. Neurology 2005;65:1504-5. 